\title{
Measuring the material properties related to human perceptions
}

Ning Pan

Department of Biological and Agricultural Engineering

University of California at Davis, CA 95616, USA

npan@ucdavis.edu

\begin{abstract}
Sensory evaluation has been widely applied in assessing the quality of many consumer products that directly serve human needs such as food, beverages, clothing, etc. This paper first examined the inherent deficiencies in this approach, due mainly to the essentially subjective nature of human sensory preference. It then argued that instinctively designating certain materials attributes as sensory perceptions is unnecessary; considering every scientific concept is in essence the processed results of our sensory organs/brains, i.e., all quantities were initially human perceptions. Scientific advances have inevitably generated gradual transformation of such human perceptions as warmth and heaviness into objective parameters measurable in physical quantities like temperature and kilograms. Then using existing successful examples, it demonstrated firmly how the sensory attributes can be assessed by more reliable instrumental methods, and envisioned the key steps to turning a perception into scientifically measurable parameter(s).
\end{abstract}

Keywords: Quality assessment; sensory attributes; human perception and bias; perception-toobjectivity transformation (POT).

\section{Sensory analysis and its inherent deficiencies}

In scientific research, being able to measure the concerned variables quantitatively and repeatedly is considered the only criterion in validating any theoretical conjectures or predictions. For example, although Peter Higgs proposed in the 1960s that broken symmetry in electroweak theory could explain the origin of mass of elementary particles, he waited until some fifty years later in 2013 to receive his shared Nobel Prize in Physics, right after the success in detection and measurement of the predicted fundamental particle by experimental physicists at CERN in 2012 1. Another well-known yet similar example has to do with the black holes. Their existence has been predicted several decades ago, following Einstein's general relativity and after rigorous analysis by some eminent physical theoreticians including Steve Hawking ${ }^{2}$. It is until the LIGO 
announcements in 2016 about the actual observations of the gravitational waves believed to be the result of interactions between black holes ${ }^{3}$, has another Nobel Prize in Physics been awarded to the scientists contributed in both theory and experimental verification ${ }^{4}$.

Even coming closer to our mundane daily activities, measured material properties in standardized units, including weight; length, time, temperature etc., have permeated into all facets of our life, as convenient and reliable indicators of the amounts and quality of the products in question. Actually, successful utilization and standardization of such numeric units have transformed our entire societal world.

However this scientific holy grail of measurement and repeatability seems to be challenged when coming to another group of materials attributes, such as those in food, clothing, beverages, perfume etc. During the use or consumption of such products, it largely relies on human sensory organs to perceive, evaluate and enjoy the performance of the products in fulfilling the intended functionalities. For instance, our eyes (vision) examine the overall appearance of a product; our ears (hearing) recognize all the acoustic information; and our nose (smelling) assesses the fragrance or odors. For edibles and beverages, we have to count on our mouth and tongue to judge the related traits. Consequently assessments of such materials attributes are done in a dramatically different way through a process termed "Sensory Analysis" - a discipline established over time ${ }^{5,6}$. "Sensory analysis (or sensory evaluation) is a scientific discipline that applies principles of experimental design and statistical analysis to the use of human senses (sight, smell, taste, touch and hearing) for the purposes of evaluating consumer products "." Like in any quantifying process, the sensory analysis followed as much as possible the proven schemes in physical sciences: it employs the human organs as the "instruments" to detect the characteristics of the object, and then converts the collected responses into numerical data. The resulted data are finally analyzed and interpreted, often against some established industrial standard references.

Given the complexities and the economic implications, standardization of the sensory analysis has been considered imperative from early on, and many industrial standards have been established regarding all important issues involved as: recruiting human assessors; products testing and responses recording; response data analysis; making inferences and interpretations about the specific products ${ }^{6}$. For instance, just in ISO Standards, Category 67.240, there are more than 40 documents regarding food sensory analysis, including the product types (dry food, 
liquor, wine, olive oil); the attribute types (taste, flavor and odor, color, texture, shelf life), methodologies (vocabularies, selection and training of assessors, sample preparation, scales use, data generation, collection and analysis). Similar documents have been issued by other authoritative standardization organizations. All such efforts however focus on largely the specific techniques and issues, not the fundamental deficiencies and possible solutions in sensory assessment.

\section{The essence of the problem}

Here is the core of the problem: on one hand, the rationality of sensory analysis couldn't be more self-evident: consumer products are to satisfy human desire or needs, it is only logical to ask real people for their judgment after actually experiencing the products. On the other hand, the faults in the process are just as clear and appealing. Using human senses as testing tools in a panel, the issues of interpersonal intrinsic bias and preference between the judges, more often than not, result in substantial deviations. Even for the same person, human senses drifts with time, mood and fatigue, etc., i.e., the scales used are shifting - just imaging the difference in food taste when starving versus sating. Also it is still impossible to define the sensation of taste, mouth feeling and smelling etc. in scientifically designated terms and units, even though there are reported cases to have methodically altered the food preferences in both human ${ }^{12}$ and rats ${ }^{13}$. Lacking the essential scientific bases and standardized terms has severely hampered the communications between parties involved in a sensory analysis, not to mention between people of different languages. Consequently, most results from sensory analysis are in relative terms and not easily applicable beyond the specific events - thus to build new knowledge based on them to advance the discipline is virtually impossible. After all, what resulted from sensory analysis is at best a "perception that may or may not represent the physical reality ${ }^{14}$. This also highlighted another dual nature of perception: first, perception depends on complex functions of several subsystems of physics, physiology and psychology, and is hence a highly sophisticated phenomenon; but in practice it presents itself simply and effortlessly because the perceiving process happens instantaneously outside our conscious awareness ${ }^{6}$.

Intellectually speaking, human perception is the organization, identification, and interpretation of sensory responses in order to represent and understand the environment ${ }^{14}$. Yet sensory perception is not the passive receipt of these signals, but is shaped by learning, memory, expectation, and attention ${ }^{14}$. Therefore, the process of sensory analysis involves diverse yet intertwining mechanisms, which roughly belong to three scientific disciplines, as shown in Fig. 1, 
i.e., Physical (including chemical, biochemical) sciences, Physiology and Psychology, with very different fundamental knowledge base and research methodologies. Intricate questions and insufficient interdisciplinary exchange and understanding have made it impossible for any singlesided attempt, in this case the sensory analysis, to identify reliable and repeatable parameters and develop the required measuring schemes in tackling such compound and multidisciplinary problems.

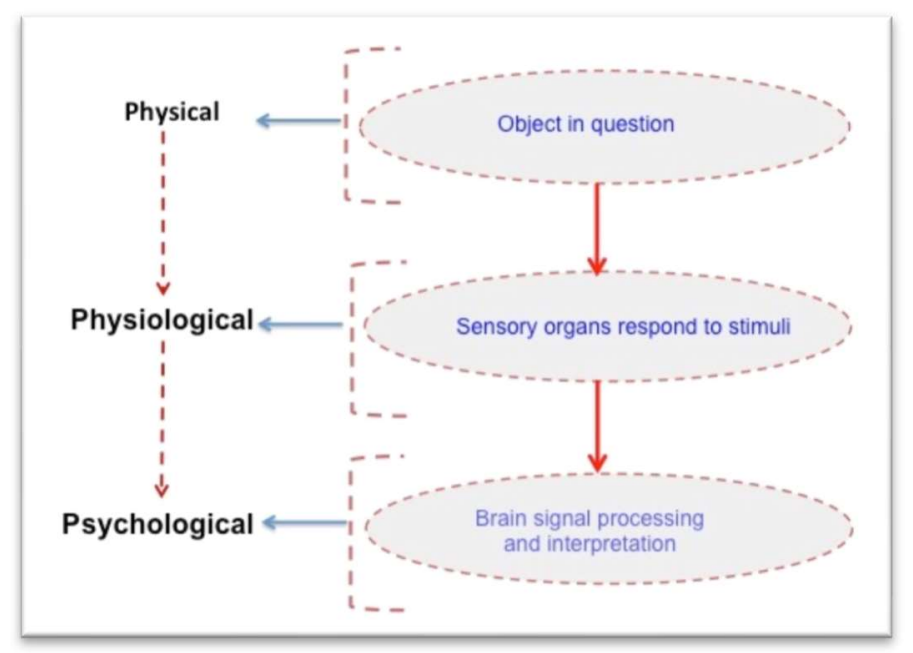

Fig. 1 Dissection of a perception formation process

Still it is highly understandable why the subjective sensory analysis has been willingly adopted by various industries; to establish a new and rigorous scientific metrics for a given product takes huge amount of work and time. Expediently, each sensory analysis scheme for a product was developed focusing on the associated sensory organ so as to form a unique set of methodology (toolbox). That is, sensory analysis has thus evolved into product-oriented, instead of scienceoriented. Such product-oriented method may appear logical and handy for industrial use in short term, and even desirable from the cultural prospective by involving information on humanity and historical evolvement. Such knowledge accumulation is counter-constructive for any further attempt from it to explore a more reliable scientific approach, and once for all.

\section{All quantities were perceptions, initially}

Perceptions are all multifaceted phenomena involving physics, physiology and psychology. Although extremely challenging in scientific frame, assessing perceptions is very important in practice for quality assurance of consumer products. In theory the most logical and solid approach in dealing with human sensory perception should be one that was built on a comprehensive framework with a thorough understanding of the mechanisms and knowledge in physics, 
physiology and psychology. This is clearly an impracticality given the current states of scientific research and knowledge accumulation in related areas. Nonetheless, if we change our viewing angle, there are reasons to be optimistic.

Although sounding a little odd, all the scientifically defined and instrumentally measurable properties today were conceived initially from human brain and hence with a subjective origin. For instance, the units used by our early ancestors to define distance were in relative terms. In the beginning, they each had in mind the relative closeness from home to different destinations. Some may view one given distance as far, and others may not. That is, the concept of distance was subjective, depending on the individual. As the need for more accuracy and quantitative-ness rose up, a "reference" length was developed and eventually became a widely accepted standard for measuring length, i.e., the unit Meter as detailed in ${ }^{15}$. But what is most intriguing is that once a numerical objective yardstick for measuring length is established and accepted, the subjectivity inherited in the original distance perception is now disappeared. $10 \mathrm{~km}$ is $10 \mathrm{~km}$ and whether it is far or not is just a personal opinion irrelevant to others. That is, by setting up a reliable, measurable numerical standard, an inquiry involving subjective perception has successfully transformed into a pure physical question.

However, if instead a route of sensory analysis were taken; since the consideration of far/near seemed to be an individual judgment, a panel would be formed, and the personal view of all relevant people on the distance between Point $A$ to Point $B$ be collected and analyzed. The process would be repeated for distance between Point $A$ to Point $C$, and so on. We would have never reached where we are today. The lessons learned from this simple example are enlightening and instructive for any attempt in measuring material properties related to human perceptions:

1. Before starting, clearly separating the physics from the perceptions involved;

2. Focusing on the physics problems only;

3. Once the physics is clarified, it would lay down a solid foundation to tackle the human perception. Very often, as in the above case, the personal preference is even no longer relevant.

From this perception-to-objectivity transformation (POT) example of distance, we can easily identify other successful cases where many once considered "human perceptions", including time, weight, and their derivatives such as frequency, area and volume, density and porosity, had gone 
through such transformation and escaped from the plaguing of the subjective bias. More interestingly, this POT process may have to be done stepwise as in the example of time and space. Einstein's general relativity "corrected" our view on such quantities so now we realized that the seemly independence between time and space was just our PERCEPTION! There are other areas where such POT has already exhibited great success - computer recognition techniques: the intelligent recognitions of face, fingerprint, language and handwriting are essentially the processes where sensory stimuli through our eyes and brains have been effectively reproduced via computer information process into reliable objective data, the same thing in the fields of virtually reality (VR) and artificial intelligence (Al).

In other words, considering that all the phenomena and concepts are in essence the processed results of our sensory organs and brains, it is highly logical to state that all quantities were initially perceptions. Although it may be a stretch to say all quantities can hence be transformed into objective ones, the sensory analysis has clearly not offered an effective solution.

\section{Objective measurement of perception-related quantities}

At this point, it may be necessary to divide all sensory problems into groups, based on the significance of the personal sensory preference:

a) Cases where sensory assessment is preferred

There are cases where POT is either unnecessary, as in music appreciation and movie viewing, or too complex to achieve soon as in issues relating to virtually reality (VR) and artificial intelligence $(\mathrm{Al})$, even though advances have been actively preceding.

b) Cases where personal preference only matters to the person

Examples include weight, length, time etc., where once succeeding in POT, the issue of subjective preference will disappear or become irrelevant to others.

c) Cases where personal preference has to be communicated to others

The cases in this group are the focus of study. Other more effective ways to deal with the perception assessment in these cases have to be sought. There are cases where individual sensory preference remains relevant, even if some necessary objective standards have been established.

Apparently, of the three types of cases, we only need to focus on Type c). In cases of this type, even some partial metrics to reflect the physical essence of the phenomena can be acquired; they will greatly reduce the complexity of the questions. For instance, in hospitals, after knowing the 
patient's instrumental test results, doctors still have to deal with the patient's personal feelings/complains; still having the objective data about the patient is hugely useful in doctor's diagnosing process. From Fig. 1, a complete sensory perceiving process includes mechanisms and issues dealt with three distinctive disciplines of physics, physiology and psychology, and why sensory analysis failed is in its attempt to lump sum all issues into a single solution, crossing all different and largely isolated disciplines.

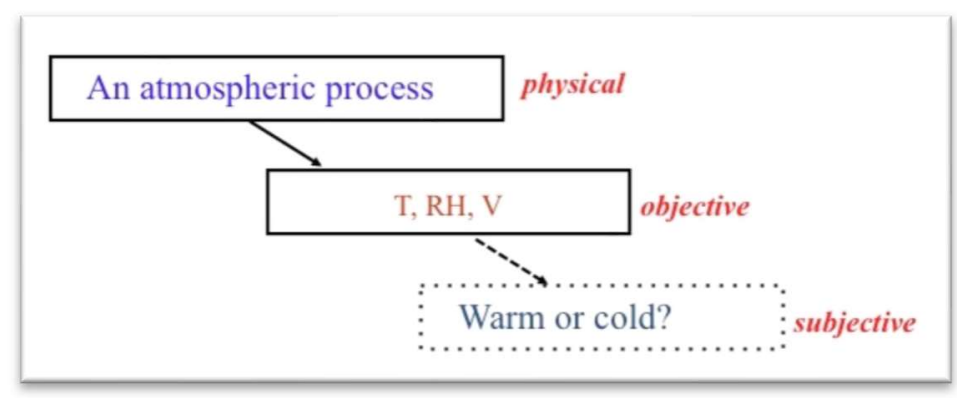

Fig. 2 Weather assessment for cloth selection

Our judgment for weather in the purpose of, e.g., clothing selection, is clearly a personal perception. However, once we developed temperature $(T)$ to specify the degree of warmth, relative humidity $(\mathrm{RH})$ for moisture content in air, wind speed $(\mathrm{V})$ to describe the rate of air movement, the weather can be described by a triad ( $T, \mathrm{RH}, \mathrm{V})$ as in Fig. 2 . The judgment for weather remains subjective, for at a given $(T, \mathrm{RH}, \mathrm{V})$ from weather report, not everyone would reach the same judgment of today's weather, desirable or undesirable. What is important though, everybody can easily make a clothing decision now and the personal bias is no longer relevant. Here is once again the enlightenment revealed from this and other examples discussed above, to succeed in conducting a subjective-to-objective transformation (POT), we should focus first and foremost on the physical reality of the problem as illustrated in Fig. 2.

As expected, there have been tremendous efforts in establishing the connection between the measurable physical properties and the human sensory response, including via chemical compositions ${ }^{16-18}$ and level of alcohol ${ }^{19}$ for wine tasting; instrument tests for perfume quality ${ }^{20}$, pasta quality ${ }^{21}$, fabric drape, softness and hand ${ }^{22-26}$. Other more advanced instruments have also been employed, such as electromyography for food texture assessment 27; gas chromatography-olfactometry (GC-O) in quality assessment of alcoholic beverages ${ }^{28}$; acoustic 
analysis in food ${ }^{29}$; fluorescence spectroscopy for dairy products ${ }^{30}$, and the electronic nose and gas chromatography-mass spectrometry for teas ${ }^{31}$ ". Such activities so far are still in the stage of exploration, and have not yet generated definite positive results. Hutchinson ${ }^{9}$ pinpointed the problem "The one thing we can do well is a lot of amazing analytical chemistry that allows us to detect a huge range of different compounds in a glass of wine. But the step we haven't got to is how that raw chemical information can be crunched together and converted into something that reflects someone's emotional response".

Still the key issues are what to measure and how to do it. A thorough examination of some successful examples above and also in the areas of color ${ }^{32}$, fabric touch/viewing ${ }^{24}$ have shed some light with generality on the potential solutions. The most important step is to identify the necessary and sufficient physical information convertible into measurable quantities - this extraction of representative and measurable quantities is THE most challenging but pivotal point to success.

This connection between sensory response and the physical properties, although inherently existent, is often indirect! Even in the simple case of weight measurement, we use the graduation readings (the length) on the balance to find (represent) the weight - a twist from weight to length is needed. In consumer products, when our concern is the thermal comfort of a wool coat, it is the cloth density, not the thermal conductivity of the wool fiber that dominates the result ${ }^{33}$. To change the drape of a skirt, we have to check the tensile and shear resistances of the cloth ${ }^{34,35}$. Such connections become more torturous in medical field: we take the reading of the mercury height level (a length) for blood pressure measurement, and, similarly, use various sensors to transform the body signs into quantities already established and measurable. Conversely, if we cannot pinpoint this intrinsic relationship, any POT attempt will escalate quickly into a mess as in many pending problems discussed before.

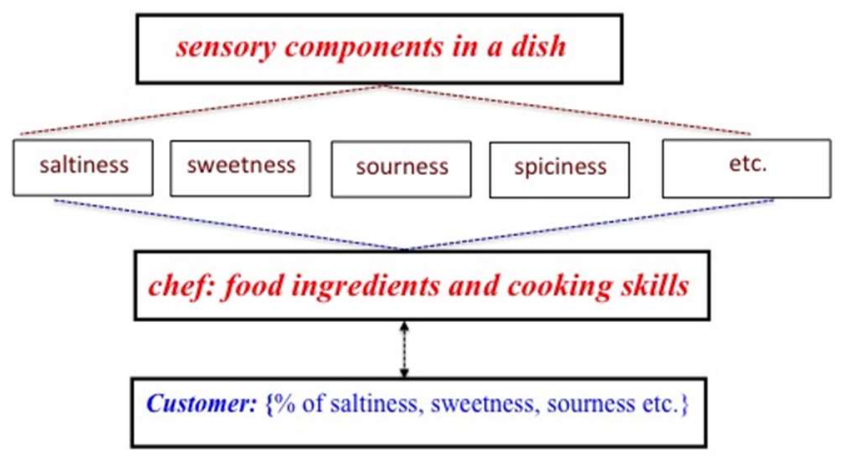


Fig. 3 New (less ambiguous) way to order your dishes

We close this paper by offering an allusion in dealing with food; e.g., dish ordering in a restaurant as illustrated in Fig. 3, just an attempt to shed some light on the problem. Ordering food is clearly a Group C type sensory process as defined above where personal preference has to be communicated to others. Nonetheless we can resolve the complexities by the following scheme:

1. Regardless the various preferences from different customers on food, food taste is ascribed to such essential and stable attributes as sweetness, saltiness, sourness, hotspiciness etc. These attributes are well defined, measurable and fully understood;

2. Next, there is already established knowledge how to fine-tune the intensity of each attribute, via e.g., increasing the amount or using more concentrated spicy, and in some cases, there is even instrument to quantify the result OBJECTIVELY. In other words, we have transferred the perception-dominated inquests into pure physical science issues;

3. Then a gourmet customer can easily communicate his/her preference to the cooker by assigning percentage or weight to each attribute.

4. A qualified cooker, already trained well how to create a given tasting constituent in terms of the types and amounts of food ingredients at certain temperature, speed, and other cooking conditions, can produce the dish to the customer desire based only on the assigned preferences to the food attributes.

During the whole process, no panel judges are involved and just one-on-one communications between individuals, and so the problem of sensory bias is no longer an obstacle!

It has to be stressed here that we are of course not proclaiming to forgo the human sensory preferences which after all are what make our life worth living! Nor we are promoting a new dish-ordering scheme. To inform people today's weather in terms of temperature, relative humidity and wind speed will definitely not, by itself, determine the full picture of a pleasant shining day! We merely demonstrate the feasibility for a much more rational and reliable approach to quantify the intrinsic physical elements embedded in sensory responses. 
To conclude, gradual transformations of human perceptions into objective parameters have been major part of scientific advances. Although it may take time in some fields, new and more effective approaches are possible besides the sensory analysis.

\section{Acknowledgement:}

This project is partly supported by US NIFA projects CA-D*-TXC-6426-RR and CA-D*-TXC-7694$\mathrm{H}$.

Ethics statement: This work did not involve any active collection of human data, but only analyses of human behavior.

Data accessibility: This work does not have any experimental data.

Competing interests: no competing financial interests.

Authors' contributions: Solo author 


\section{References Cited:}

1 Aad, G. et al. Observation of a new particle in the search for the Standard Model Higgs boson with the ATLAS detector at the LHC. Phys. Lett. B 716, 1-29, doi:10.1016/j.physletb.2012.08.020 (2012).

2 Hawking, S. W. Particle Creation By Black-Holes. Commun. Math. Phys. 43, 199-220, doi:10.1007/bf02345020 (1975).

3 Collaboration, L. S. et al. Observation of Gravitational Waves from a Binary Black Hole Merger. Physical Review Letters 116, 061102 (2016).

$4 \quad$ The Nobel Prize in Physics 2017, <https://www.nobelprize.org/nobel prizes/physics/laureates/2017/> (2017).

5 Stone, H. \& Sidel, J. L. Sensory Evaluation Practices. 3rd edn, (Elsevier Inc., 2004).

6 Morten C. Meilgaard, Gail Vance Civille \& Carr, B. T. Sensory Evaluation Techniques. 5th edn, (CRC Press, 2016).

$7 \quad$ Peynaud, E. The Taste of Wine: The Art and Science of Wine Appreciation. 2nd edn, (John Wiley \& Sons, Inc.,, 1996).

8 Contributors2, W. Wine tasting, $<$ https://en.wikipedia.org/w/index.php?title=Wine tasting\&oldid=760807645> (2014).

9 Derbyshire, D. in The Guardian (22 June 2013).

10 Hodgson, R. An Examination of Judge Reliability at a major U.S. Wine Competition. Journal of Wine Economics 3, 105-113 (2008).

11 Finewine, M. s. White Wine Aroma Wheel, <http://merchantsfinewine.com/faq-items/wine-aromawheels/> (2013).

12 Berthoud, H. R. \& Zheng, H. Modulation of taste responsiveness and food preference by obesity and weight loss. Physiol. Behav. 107, 527-532 (2012).

13 Sandoval, D. A. \& Seeley, R. J. Physiology: Gut feeling for food choice. Nature advance online publication, doi:10.1038/nature21499 (2017).

14 Schacter, D. L., Gilbert, D. T., Wegner, D. M. \& Nock, M. K. Psychology. 3rd edn, (Worth Publishers, 2014).

15 Contributors, W. Introduction to the metric system, $<$ https://en.wikipedia.org/w/index.php?title=Introduction to the metric system\&oldid=766536450 $>$ (2017). Bosenko, 0. \& Eder, R. Correlations between the chemical composition and objective or subjective colour quality of red wines. Mitteilungen Klosterneuburg 61, 166-178 (2011).

17 Hopfer, H., Ebeler, S. E. \& Heymann, H. The Combined Effects of Storage Temperature and Packaging Type on the Sensory and Chemical Properties of Chardonnay. Journal of Agricultural and Food Chemistry 60, 10743-10754, doi:10.1021/jf302910f (2012).

18 Buffon, P., Heymann, H. \& Block, D. E. Sensory and Chemical Effects of Cross-Flow Filtration on White and Red Wines. American Journal of Enology and Viticulture 65, 305-314, doi:10.5344/ajev.2014.13090 (2014).

19 King, E. S., Dunn, R. L. \& Heymann, H. The influence of alcohol on the sensory perception of red wines. Food Quality and Preference 28, 235-243, doi:10.1016/j.foodqual.2012.08.013 (2013).

20 Korifi, R., Le Dreau, Y., Antinelli, J. F., Valls, R. \& Dupuy, N. CIEL*a*b* color space predictive models for colorimetry devices - Analysis of perfume quality. Talanta 104, 58-66, doi:10.1016/j.talanta.2012.11.026 (2013).

21 Cole, M. E. Review - prediction and measurement of pasta quality. International Journal of Food Science and Technology 26, 133-151 (1991).

22 Chu, C. C., Cummings, C. L. \& Teixeira, N. A. Mechanics of elastic performance of textile materials .5. A study of the factors affecting the drape of fabrics - the development of a drape meter. Textile Research Journal 20, 539-548, doi:10.1177/004051755002000802 (1950).

23 Kawabata, S. The Standardization and Analysis of Handle Evaluation. 2nd edn, (The Textile Machinery Society of Japan, 1980).

24 Pan, N. Quantification and evaluation of human tactile sense towards fabrics. International Journal of Design and Nature 1, 48-60 (2006).

25 Holmes, D. P. \& Crosby, A. J. Draping Films: A Wrinkle to Fold Transition. Physical Review Letters 105, doi:10.1103/PhysRevLett.105.038303 (2010). 
26 Cao, Y. P. \& Hutchinson, J. W. From wrinkles to creases in elastomers: the instability and imperfection-sensitivity of wrinkling. Proceedings of the Royal Society a-Mathematical Physical and Engineering Sciences 468, 94-115, doi:10.1098/rspa.2011.0384 (2012).

27 Gonzalez, R., Montoya, I. \& Carcel, J. Review: The use of electromyography on food texture assessment. Food Science and Technology International 7, 461-471, doi:10.1106/nrht-l39d-hy1y-8rgb (2001).

28 Plutowska, B. \& Wardencki, W. Application of gas chromatography-olfactometry (GC-0) in analysis and quality assessment of alcoholic beverages - A review. Food Chemistry 107, 449-463, doi:10.1016/j.foodchem.2007.08.058 (2008).

29 Aboonajmi, M., Jahangiri, M. \& Hassan-Beygi, S. R. A review on application of acoustic analysis in quality evaluation of agro-food products. Journal of Food Processing and Preservation 39, 3175-3188, doi:10.1111/jfpp.12444 (2015).

30 Andersen, C. M. \& Mortensen, G. Fluorescence spectroscopy: A rapid tool for analyzing dairy products. Journal of Agricultural and Food Chemistry 56, 720-729, doi:10.1021/jf072025o (2008).

31 Qin, Z. H. et al. Evaluation of Chinese tea by the electronic nose and gas chromatography-mass spectrometry: Correlation with sensory properties and classification according to grade level. Food Research International 53, 864-874, doi:10.1016/j.foodres.2013.02.005 (2013).

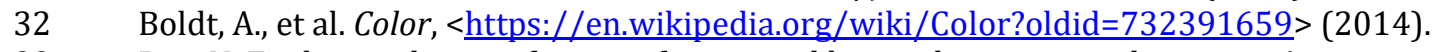

33 Pan, N. Exploring the significance of structural hierarchy in material systems-A review. Applied Physics Reviews 1, doi:10.1063/1.4871365 (2014).

34 Peirce, F. T. The handle of cloth as a measurable quality. J. Text. Inst. 28, T45 (1937).

35 Hearle, J. W. S., Grosberg, P. \& Backer, S. Structural Mechanics of Yarns and Fabrics. (WileyInterscience, 1969). 